\title{
Non-orientable Genus of a Knot in Punctured $\mathrm{C} P^{2}$
}

\author{
Kouki SATO and Motoo TANGE \\ Tokyo Institute of Technology and University of Tsukuba \\ (Communicated by Y. Kuno)
}

\begin{abstract}
For a closed 4-manifold $X$, any knot $K$ in the boundary of punctured $X$ bounds a non-orientable and null-homologous embedded surface in punctured $X$. Thus we can define an invariant $\gamma_{X}^{0}(K)$ to be the smallest first Betti number of such surfaces. Note that $\gamma_{S^{4}}^{0}$ is equal to the non-orientable 4-ball genus. While it is very likely that for a given $X, \gamma_{X}^{0}$ has no upper bound, it is difficult to show it. Recently, Batson showed that $\gamma_{S^{4}}^{0}$ has no upper bound. In this paper we show that for any positive integer $n, \gamma_{n \mathbf{C} P^{2}}^{0}$ has no upper bound.
\end{abstract}

\section{Introduction}

Throughout this paper, we assume that all manifolds and embedding dealt in this paper are smooth. Moreover, we assume that all 4-manifolds are orientable, oriented and simplyconnected, and all surfaces are compact. If $X$ is a closed 4-manifold, punc $X$ denotes $X$ with an open 4-ball deleted.

Let $X$ be a closed 4-manifold and $K$ a knot in $\partial$ (punc $X$ ). We say that $K$ bounds $F$ in $\partial$ (punc $X$ ) if $F$ is a surface embedded in punc $X$ with boundary $K$. For a given 4-manifold $X$ and a second homology class of punc $X$, the set which consists of the diffeomorphism types of embedding surfaces representing the class and that $K$ bounds, is a significant invariant of the isotopy type of $K$. In the simplest case that $X=S^{4}$ and the embedded surfaces are all restricted to orientable surfaces, such an invariant has been studied as 4-ball genus $g_{4}$ by many topologists. For a knot $K$ in $\partial($ punc $X) \cong S^{3}$, it is natural to ask which kinds of surfaces $K$ can bound.

In this paper, we focus on non-orientable surfaces embedded in punc $X$ with boundary $K$. It is known that for any homology class $\xi \in H_{2}\left(\operatorname{punc} X, \partial(\operatorname{punc} X) ; \mathbf{Z}_{2}\right)$ and any knot $K$ in $\partial$ (punc $X), K$ bounds a non-orientable surface which represents $\xi$. Hence we can define $\gamma_{X}(K, \xi)$ to be the smallest first Betti number of any non-orientable surface embedded in punc $X$ with boundary $K$ which represents $\xi$. In particular, we investigate the smallest number

$$
\gamma_{X}(K):=\min \left\{\gamma_{X}(K, \xi) \mid \xi \in H_{2}\left(\operatorname{punc} X, \partial(\operatorname{punc} X) ; \mathbf{Z}_{2}\right)\right\}
$$


and $\gamma^{0}(K):=\gamma_{X}(K, 0)$ in this paper, since they can be defined for any 4-manifold $X$ and characterize $X$ from the viewpoint of knot theory. Moreover, both $\gamma_{S^{4}}(K)$ and $\gamma_{S^{4}}^{0}(K)$ are equal to the non-orientable 4-ball genus $\gamma_{4}(K)$, which is the smallest first Betti number of any non-orientable surface embedded in $B^{4}$ with boundary $K$. Hence $\gamma_{X}(K)$ and $\gamma_{X}^{0}(K)$ are generalizations of $\gamma_{4}(K)$.

While $\gamma_{4}$ has been investigated since 1975 [9], it is still a difficult problem to evaluate $\gamma_{4}$. In fact, it had been unknown whether or not $\gamma_{4}$ has an upper bound until recently. An excellent reference for related studies is [2]. In 2012, Batson proved that $\gamma_{4}$ has no upper bound by establishing the following inequality.

THEOREM 1 ([1]). Let $K \subset S^{3}$ be a knot. Then

$$
\gamma_{4}(K) \geq \frac{-\sigma(K)}{2}+d\left(S_{1}^{3}(K)\right)
$$

where $\sigma(K)$ denotes the signature of $K$ and $d\left(S_{1}^{3}(K)\right)$ the Heegaard-Floer d-invariant of the 1 -surgery along $K$.

The definition of the $(p / q)$-surgery $S_{p / q}^{3}(K)$ along $K$ will be given at the last of this section.

In particular, Batson showed that $\gamma_{4}\left(T_{2 k, 2 k-1}\right)=\gamma_{4}\left(T_{-2 k, 2 k-1}\right)=k-1$ for any positive integer $k$, where $T_{p, q}$ denotes the right handed $(p, q)$-torus knot.

On the other hand, we can see that $T_{-2 k, 2 k-1}$ bounds a null-homologous embedded Möbius band in punc $\mathbf{C} P^{2}$ as follows. We first consider a Möbius band properly embedded in $B^{4}$ with boundary the unknot. Then the boundary of this surface can be deformed to $T_{2 k, 1}$ by an isotopy. After attaching the $(+1)$-framed 2-handle and handle sliding as in Figure 1 , we have the desired surface in punc $\mathbf{C} P^{2}$. Note that $T_{2 k, 2 k-1}$ bounds a null-homologous embedded Möbius band in punc $\overline{\mathbf{C} P^{2}}$. This fact implies that $\gamma_{X}$ and $\gamma_{X}^{0}$ largely depend on the choice of $X$.

In this paper, we consider the existence problem of upper bounds of non-orientable $X$ genera for a general 4-manifold $X$.
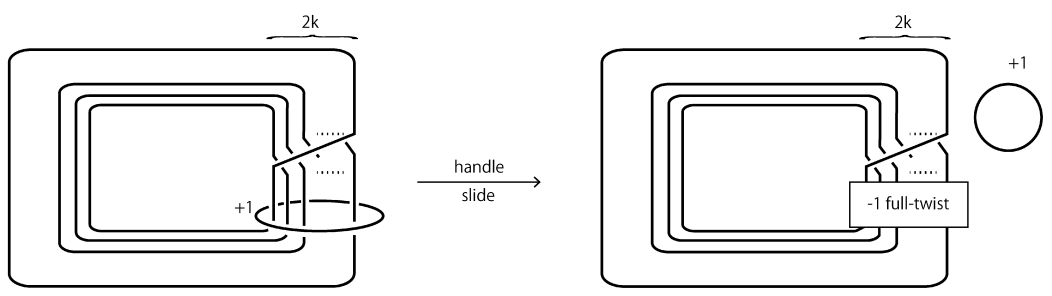

FIGURE 1. $T_{-2 k, 2 k-1}$ bounds a null-homologous embedded Möbius band in punc $\mathbf{C} P^{2}$. 
PROBLEM 1. For a given 4-manifold $X$, do $\gamma_{X}$ and $\gamma_{X}^{0}$ have upper bounds?

In the case of $\gamma_{X}$, it is known that there exist infinitely many 4-manifolds which give the affirmative answer of Problem 1. In fact, Suzuki [8] and Norman [3] proved that if $X$ is diffeomorphic to $S^{2} \times S^{2}$ or $\mathbf{C} P^{2} \# \overline{\mathbf{C} P^{2}}$, then any knot bounds an embedded disk $D$ in punc $X$. Moreover, by taking the connected sum of (punc $X, D),\left(S^{4}, \mathbf{R} P^{2}\right)$ and the pair $(N, \varnothing)$ of any closed 4-manifold $N$ and the empty set, we see that any knot which bounds an embedded disk in punc $X$ also bounds an embedded Möbius band in punc $(X \# N)$. This implies that if $X$ is diffeomorphic to $\left(S^{2} \times S^{2}\right) \# N$ or $\mathbf{C} P^{2} \# \overline{\mathbf{C} P^{2}} \# N$ for a closed 4-manifold $N$, then the value of $\gamma_{X}$ is always 1 . We note that these results have been restricted to indefinite 4-manifolds (i.e., 4-manifolds with indefinite intersection forms).

Now, if $X$ is a definite 4-manifold, then do $\gamma_{X}$ and $\gamma_{X}^{0}$ have upper bounds? In this paper, we give the negative answer for Problem 1 in the case of $\gamma_{n \mathbf{C} P^{2}}^{0}$.

THEOREM 2. For any $n, k \in \mathbf{N}$, there exists a knot $K$ such that $\gamma_{n \mathbf{C} P^{2}}^{0}(K)=k$.

We prove Theorem 2 by extending Batson's inequality to the case of $\gamma_{n \mathbf{C} P^{2}}^{0}$ as follows.

THEOREM 3. Let $K \subset S^{3}$ be a knot and $n \in \mathbf{N}$. Then we have

$$
\gamma_{n \mathbf{C} P^{2}}^{0}(K) \geq \frac{-\sigma(K)}{2}+d\left(S_{1}^{3}(K)\right)-n .
$$

Moreover, since $-\sigma\left(T_{2 k, 2 k-1}\right)=\sigma\left(T_{-2 k, 2 k-1}\right)=2 k^{2}-2, d\left(S_{1}^{3}\left(T_{2 k, 2 k-1}\right)\right)=-k^{2}+k$ and $d\left(S_{1}^{3}\left(T_{-2 k, 2 k-1}\right)\right)=0$, it follows that

$$
\gamma_{\mathbf{C} P^{2}}^{0}\left(T_{2 k, 2 k-1}\right) \geq k-2 \text { and } \gamma_{\mathbf{C} P^{2}}^{0}\left(T_{-2 k, 2 k-1}\right)=1 .
$$

On the other hand, one should compare these conditions with the equalities

$$
\gamma_{4}\left(T_{2 k, 2 k-1}\right)=\gamma_{4}\left(T_{-2 k, 2 k-1}\right)=k-1 .
$$

Here we define (Dehn) $(p / q)$-surgery along a knot $K$ in $S^{3}$. Let $v(A)$ denote the tubular neighborhood of a submanifold $A$. We define the $p / q$-surgery along a knot $K$ in $S^{3}$ to be the following 3-manifold

$$
S_{p / q}^{3}(K):=\left[S^{3}-v(K)\right] \cup\left(D^{2} \times S^{1}\right)
$$

which is obtained by removing the neighborhood of $K$ and by gluing one solid torus along the both boundaries. The gluing map is defined to be

$$
\partial D^{2} \times\{\mathrm{pt}\} \rightarrow q \cdot \lambda+p \cdot \mu,
$$

where $\mu$ and $\lambda$ are the meridian and longitude of $K$. The notation $q \cdot \lambda+p \cdot \mu$ stands for a simple closed curve on the torus whose homology class is $q[\lambda]+p[\mu]$. 


\section{A short review of the Heegaard Floer theory}

In this section we give a brief review of the definition of Heegaard-Floer $d$-invariant, and some results which are employed in the present paper.

Let $(Y, \mathfrak{s})$ be an oriented closed 3-manifold associated with a $\operatorname{Spin}^{c}$ structure $\mathfrak{s}$. We call such a pair $(Y, \mathfrak{s})$ a $\operatorname{Spin}^{c}$ 3-manifold. Ozsváth and Szabó in [4] defined the Heegaard Floer homologies $H F^{*}(Y, \mathfrak{s}) \quad(*=+,-, \infty)$ for any $\operatorname{Spin}^{c} 3$-manifold $(Y, \mathfrak{s})$. If the 1 st Chern class of $\mathfrak{s}$ is a torsion element, then the Heegaard Floer homologies become absolutely Q-graded $\mathbf{F}[U] \otimes\left(H_{1}(Y) /\right.$ Tors $)$-modules, where $U$ is the action decreasing the grading by 2. Throughout this paper, we consider the coefficient field $\mathbf{F}$ of all homologies as the field with $\operatorname{char}(\mathbf{F})=2$. These homology groups are related to one another by the following exact sequence:

$$
\cdots \rightarrow H F^{-}(Y, \mathfrak{s}) \rightarrow H F^{\infty}(Y, \mathfrak{s}) \stackrel{\pi}{\rightarrow} H F^{+}(Y, \mathfrak{s}) \rightarrow H F^{-}(Y, \mathfrak{s}) \rightarrow \cdots
$$

Let $Y$ be a rational homology 3-sphere. The (Heegaard-Floer) $d$-invariant $d(Y, \mathfrak{s})$ is defined to be the minimal grading of the image of the map $\pi$ and the value is a rational number. The one component of the map $\pi$ is isomorphic to $\mathbf{F}\left[U, U^{-1}\right] /(U \cdot \mathbf{F}[U])$ and we denote it by $\mathcal{T}_{(d)}^{+}$, where $d$ is the minimal grading of the component. If $Y$ is an integer homology 3-sphere, then $Y$ has a unique $\operatorname{Spin}^{c}$ structure. In such a case, we denote the $d$-invariant simply by $d(Y)$ and the value of the invariant becomes an even integer.

Let $(Y, \mathfrak{s})$ be a $\operatorname{Spin}^{c} 3$-manifold with a torsion $\operatorname{Spin}^{c}$ structure. Let $d_{b}(Y, \mathfrak{s})$ denote the bottom-most $d$-invariant, i.e., the minimal grading of the image of $\pi$ in the kernel of the ( $H_{1}(Y) /$ Tors)-action. Then, the following theorem follows. Here $\beta_{i}$ is the $i$-th Betti number and $\beta_{2}^{+}(X)$ (or $\beta_{2}^{-}(X)$ ) is the number of positive (or negative respectively) eigenvalues of the intersection form on $H_{2}(X)$. A $\operatorname{Spin}^{c} 3$-manifold $(Y, \mathfrak{s})$ with a torsion $\operatorname{Spin}^{c}$ structure is said to have standard $H F^{\infty}$ if $H F^{\infty}(Y, \mathfrak{s})$ is isomorphic to $\mathcal{T}^{\infty}:=\left(\Lambda^{*} H^{1}(Y, \mathbf{Z})\right) \otimes \mathbf{F}\left[U, U^{-1}\right]$.

THEOREM 4 ([4]). Let $(Y, \mathfrak{t})$ be a Spin ${ }^{c} 3$-manifold (not necessarily connected) with a torsion Spin $^{c}$ structure which has standard $H F^{\infty}$. If $X$ is a negative semi-definite 4-manifold with boundary $Y$ such that the restriction map $H^{1}(X ; \mathbf{Z}) \rightarrow H^{1}(Y ; \mathbf{Z})$ is trivial, and $\mathfrak{s}$ is a Spin $^{c}$ structure on $X$ restricting to $\mathfrak{t}$ on $Y$, then

$$
c_{1}(\mathfrak{s})^{2}+\beta_{2}^{-}(X) \leq 4 d_{b}(Y, \mathfrak{t})+2 \beta_{1}(Y) .
$$

Let $K$ be a knot in $S^{3}$. In [5] the double complex $\left(C F K^{\infty}\left(S^{3}, K\right), \partial^{\infty}\right)$ with coordinates $i, j$ is defined to be a filtered chain complex of $C F^{\infty}\left(S^{3}\right)$ associated with $K$ in $S^{3}$. It is called knot Floer chain complex, and its homology group is called knot Floer homology. In this paper, we often omit $S^{3}$ in $C F K^{\infty}\left(S^{3}, K\right)$. The filtered chain homotopy type is a knot isotopy invariant. For the knot Floer homology, we use the same notations as the ones in [5]. We also use the notation $\partial^{\infty}$ for the differentials even for restricted or quotient complexes of $C F K^{\infty}$.

We introduce the following proposition and formula for a sufficiently large integer $p$ : 
Proposition 1 ([5]). For a sufficiently large integer $p$, we have the following isomorphism

$$
H F_{\ell}^{+}\left(S_{p}^{3}(K),[0]\right) \cong H_{k}\left(C F K^{\infty}\{i \geq 0 \text { or } j \geq 0\}\right),
$$

where $\ell=k+(p-1) / 4$.

In particular, we have

$$
d\left(S_{1}^{3}(K)\right)=\tilde{d}\left(S_{p}^{3}(K),[0]\right),
$$

where $\tilde{d}$ is the unshifted $d$-invariant, i.e., $\tilde{d}\left(S_{p}^{3}(K),[0]\right)=d\left(S_{p}^{3}(K),[0]\right)-(p-1) / 4$.

\section{Extension of Batson's inequality}

In order to prove Theorem 3, we first prove the following proposition.

Proposition 2. Let $K \subset \partial\left(\operatorname{punc}\left(n \overline{\mathbf{C} P^{2}}\right)\right)$ be a knot and $F \subset \operatorname{punc}\left(n \overline{\mathbf{C} P^{2}}\right)$ a nonorientable embedded surface with boundary $K$. Then

$$
\beta_{1}(F) \geq \frac{e(F)}{2}-2 d\left(S_{-1}^{3}(K)\right) .
$$

Batson showed in [1, Theorem 4] that this inequality holds for the case where $n=0$; that is, $F \subset B^{4}$. Hence this proposition is an extension of [1, Theorem 4].

In Proposition 2, $e(F)$ is the normal Euler number of $F$ defined as follows. Let $X$ be a closed 4-manifold and $F$ a properly embedded surface in punc $X$ with $\partial F \cong S^{1}$. Take an orientation of $\partial F$ and a section $\tilde{F}$ of the normal bundle of $F$ that does not intersect $F$. Let $e(F)=-\operatorname{lk}(\partial F, \partial \tilde{F})$, where the orientation of $\partial \tilde{F}$ is induced from $\partial F$. Note that $e(F)$ does not depend on the choice of the orientation for $\partial F$. We call $e(F)$ the normal Euler number of $F$ (see [10]). We also note that if $F$ is orientable, then $e(F)$ is equal to the self-intersection number of $F$.

In order to prove Proposition 2, we need the following lemma. Let $X$ be a closed 4-manifold and $K \subset \partial$ (punc $X)$ a knot. We identify $H_{2}(X ; \mathbf{Z}) \oplus H_{2}\left(S^{2} \times S^{2} ; \mathbf{Z}\right)$ with $H_{2}\left(\operatorname{punc}\left(X \#\left(S^{2} \times S^{2}\right)\right), \partial\left(\operatorname{punc}\left(X \#\left(S^{2} \times S^{2}\right)\right)\right) ; \mathbf{Z}\right)$.

LEMMA 1. For any non-orientable embedded surface $F \subset$ punc $X$ with boundary $K$ and odd $\beta_{1}$, there exists an orientable embedded surface $F^{\prime} \subset \operatorname{punc}\left(X \#\left(S^{2} \times S^{2}\right)\right)$ with boundary $K$ which satisfies

1. $\beta_{1}\left(F^{\prime}\right)=\beta_{1}(F)-1$,

2. $e\left(F^{\prime}\right)=e(F)+2$, and

3. $\left[F^{\prime}, \partial F^{\prime}\right]=v \oplus(2 \alpha+b \beta)$ for some $v \in H_{2}(X ; \mathbf{Z})$ and $b \in \mathbf{Z}$.

Here $\alpha$ and $\beta$ are standard generators of $H_{2}\left(S^{2} \times S^{2} ; \mathbf{Z}\right)$ such that $\alpha \cdot \alpha=\beta \cdot \beta=0$, and $\alpha \cdot \beta=1$. 


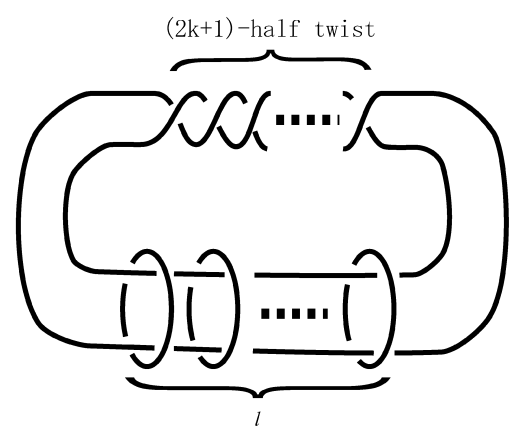

FiguRE 2. Our link $L$.

Proof. Since $\beta_{1}(F)$ is odd, there exists a simple closed curve $C$ in $F$ whose tubular neighborhood in $F$ is diffeomorphic to the Möbius band and $F \backslash C$ is orientable. Since punc $X$ is simply-connected, $C$ is null-homotopic in $X$. Moreover, every homotopy may be replaced with an isotopy in these dimensions, and hence $C$ is isotopic to a trivial circle. This implies that $C$ bounds an embedded disk $D$ in punc $X$. We can assume that $D$ is transverse to $F$ in the interior of $D$. Then $F \cap D$ consists of $C$ and finitely many points $\left\{p_{i}\right\}(i=1,2, \ldots, l)$. Moreover, $v(D)$ is diffeomorphic to $D \times D^{2}$, and $F \cap v(D)$ consists of a Möbius band properly embedded in $\partial D \times D^{2}$ and $l$ 2-disks $p_{i} \times D^{2}$. This implies that $L:=\partial(F \cap \nu(D)) \subset \partial v(D)$ is a link as in Figure 2. In the same way as Step 3 and Step 4 in the proof of [1, Proposition 1.4], we can verify that $L$ bounds $l+1$ embedded disks $E$ in $\operatorname{punc}\left(S^{2} \times S^{2}\right)$ which satisfy $e(E)=e(F \cap v(D))+2$. Finally, by removing $v(D)$ from punc $X$ and gluing $\left(\operatorname{punc}\left(S^{2} \times S^{2}\right)\right.$, $E)$ along $(\partial v(D), L)$, we obtain a new orientable embedded surface $F^{\prime}$ in punc $\left(X \#\left(S^{2} \times S^{2}\right)\right)$. It is easy to check that $F^{\prime}$ satisfies the above conditions from (1) to (3).

We next prove the following lemma, which is a generalization of a discussion in [1, Section 4].

LEMMA 2. Let $M$ be an integer homology 3-sphere, $X$ a simply-connected 4-manifold such that $\partial X=M$ and $\beta_{2}^{+}(X)=1$ and $\Sigma$ an orientable closed surface embedded in $X$ with genus $g$ and self-intersection $m$. Then for any Spin ${ }^{c}$ structure $\mathfrak{s}$ on $X$ which satisfies $\left\langle c_{1}(\mathfrak{s}),[\Sigma]\right\rangle=m-2 g>0$, the following inequality holds:

$$
c_{1}(\mathfrak{s})^{2}+\beta_{2}^{-}(X) \leq 1+4 d(M) .
$$

Proof. Let $X^{\prime}$ be the complement $X \backslash v(\Sigma)$. Then $X^{\prime}$ is a negative semi-definite 4manifold with disconnected boundaries $Y_{g,-m} \amalg M$, where $Y_{g,-m}$ denotes the Euler number $-m$ circle bundle over $\Sigma$.

We prove that $X^{\prime}$ is negative semi-definite. Let $n\left(X^{\prime}\right)$ denote the number of zero eigenvectors in the intersection form $Q_{X^{\prime}}$. We can verify from elementary homology theory that 
$\beta_{2}\left(X^{\prime}\right)=\beta_{2}(X)+2 g-1=\beta_{2}^{-}(X)+2 g$. Furthermore, by Novikov's additivity formula, $\sigma\left(X^{\prime}\right)=\sigma(X)-\sigma(v(\Sigma))=-\beta_{2}^{-}(X)$. Thus we have $2 \beta_{2}^{+}\left(X^{\prime}\right)+n\left(X^{\prime}\right)=2 g$, and $\beta_{2}^{+}\left(X^{\prime}\right) \leq 0$ is equivalent to $n\left(X^{\prime}\right) \geq 2 g$. The homology exact sequence of the pair $\left(X^{\prime}, Y_{g,-m}\right)$ shows the following exact sequence:

$$
H_{2}\left(X^{\prime} ; \mathbf{Q}\right) \rightarrow H_{2}\left(X^{\prime}, Y_{g,-m} ; \mathbf{Q}\right) \stackrel{\text { surj. }}{\rightarrow} H_{1}\left(Y_{g,-m} ; \mathbf{Q}\right) \cong \mathbf{Q}^{2 g}
$$

and it implies $n\left(X^{\prime}\right) \geq 2 g$.

We apply Theorem 4 to the tuple $\left(X^{\prime}, Y_{g,-m} \amalg M,\left.\mathfrak{s}\right|_{X^{\prime}}\right)$. The standard-ness of $Y_{g,-m} \amalg M$ is described in the proof of [1, Theorem 1.5]. Moreover, we can verify in the same way as [1, Section 4] that $H^{1}\left(X^{\prime} ; \mathbf{Z}\right)=0$ and the image of the restriction map $H^{2}(v(\Sigma) ; \mathbf{Z}) \rightarrow$ $H^{2}\left(Y_{g,-m} ; \mathbf{Z}\right)$ is a torsion group. This implies that for any $\operatorname{Spin}^{c}$ structure on $X$, the restricted $\operatorname{Spin}^{c}$ structure on $Y_{g,-m}$ is a torsion $\operatorname{Spin}^{c}$ structure. Thus, it follows that the tuple $\left(X^{\prime}, Y_{g,-m} \amalg M,\left.\mathfrak{s}\right|_{X^{\prime}}\right)$ satisfies all conditions of Theorem 4.

By Theorem 4, we have

$$
c_{1}\left(\left.\mathfrak{s}\right|_{X^{\prime}}\right)^{2}+\beta_{2}^{-}\left(X^{\prime}\right) \leq 4 d_{b}\left(Y_{g,-m},\left.\mathfrak{s}\right|_{Y_{g,-m}}\right)+4 d(M)+2 \beta_{1}\left(Y_{g,-m}\right) .
$$

Let us compute each term in the inequality (2). In order to compute $c_{1}\left(\left.\mathfrak{s}\right|_{X^{\prime}}\right)^{2}$, we decompose the intersection form of $X$ in terms of the Q-valued intersection forms on $v(\Sigma)$ and $X^{\prime}$; if $c \in H^{2}(X)$, then

$$
Q_{X}(c)=Q_{\nu(\Sigma)}\left(\left.c\right|_{\nu(\Sigma)}\right)+Q_{X^{\prime}}\left(\left.c\right|_{X^{\prime}}\right) .
$$

This gives $c_{1}(\mathfrak{s})^{2}=c_{1}\left(\left.\mathfrak{s}\right|_{v(\Sigma)}\right)^{2}+c_{1}\left(\left.\mathfrak{s}\right|_{X^{\prime}}\right)^{2}$. Hence we have

$$
c_{1}\left(\left.\mathfrak{s}\right|_{X^{\prime}}\right)^{2}=c_{1}(\mathfrak{s})^{2}-c_{1}\left(\left.\mathfrak{s}\right|_{\nu(\Sigma)}\right)^{2}=c_{1}(\mathfrak{s})^{2}-\frac{(m-2 g)^{2}}{m} .
$$

For the above $\operatorname{Spin}^{c}$ structure $\left.\mathfrak{s}\right|_{Y_{g,-m}}$, the $d$-invariant of $Y_{g,-m}$ is computed in [4, Section 9]. If $\left\langle c_{1}(\mathfrak{s}),[\Sigma]\right\rangle=m-2 g>0$, then

$$
d_{b}\left(Y_{g,-m},\left.\mathfrak{s}\right|_{Y_{g,-m}}\right)=\frac{1}{4}-\frac{g^{2}}{m}-\frac{m}{4} .
$$

The substitution of all the values computed above reduces (2) to

$$
c_{1}(\mathfrak{s})^{2}-\frac{(m-2 g)^{2}}{m}+\beta_{2}^{-}\left(X^{\prime}\right) \leq 4\left(\frac{1}{4}-\frac{g^{2}}{m}-\frac{m}{4}\right)+4 d(M)+2(2 g) .
$$

Since $\beta_{2}^{-}\left(X^{\prime}\right)=\beta_{2}^{-}(X),(3)$ gives the inequality

$$
c_{1}(\mathfrak{s})^{2}+\beta_{2}^{-}(X) \leq 1+4 d(M) .
$$


Proof of Proposition 2. Note that for any knot $K, d\left(S_{-1}^{3}(K)\right) \geq 0$. Hence in the case that $e(F) \leq \beta_{1}(F)$, it is clear that this proposition holds. Therefore we assume that $e(F)>\beta_{1}(F)$.

We first give the proof for the case where $\beta_{1}(F)$ is odd. By applying Lemma 1 to $F \subset$ $\operatorname{punc}\left(n \mathbf{C} P^{2}\right)$, we obtain an orientable embedded surface $F^{\prime} \subset \operatorname{punc}\left(n \overline{\mathbf{C} P^{2}} \#\left(S^{2} \times S^{2}\right)\right)$ with boundary $K$ whose homology class is

$$
\left[F^{\prime}, \partial F^{\prime}\right]=\sum_{i=1}^{j} 2 a_{i} \bar{\gamma}_{i}+\sum_{i=j+1}^{n}\left(2 a_{i}+1\right) \bar{\gamma}_{i}+2 \alpha+b \beta \quad\left(a_{i}, j \in \mathbf{Z}, 0 \leq j \leq n\right),
$$

where $\bar{\gamma}_{i}(i=1, \ldots, n)$ are standard generators of $H_{2}\left(n \overline{\mathbf{C} P^{2}} ; \mathbf{Z}\right)$ such that $\bar{\gamma}_{i} \cdot \bar{\gamma}_{j}=-\delta_{i j}$ (Kronecker's delta). Without loss of generality, we may permutate the order of $\bar{\gamma}_{i}$.

Since $F^{\prime}$ is orientable, we have

$$
e\left(F^{\prime}\right)=\left[F^{\prime}, \partial F^{\prime}\right] \cdot\left[F^{\prime}, \partial F^{\prime}\right]=-\sum_{i=1}^{j} 4 a_{i}^{2}-\sum_{i=j+1}^{n}\left(2 a_{i}+1\right)^{2}+4 b .
$$

Attaching a (-1)-framed 2-handle along $K$, we have a 4-manifold $\bar{W}$ with boundary $S_{-1}^{3}(K)$ and the intersection form

$$
Q_{\bar{W}}=\left(\begin{array}{c|ccc|cc}
-1 & 0 & \ldots & 0 & 0 & 0 \\
\hline 0 & -1 & & O & 0 & 0 \\
\vdots & & \ddots & & \vdots & \vdots \\
0 & O & & -1 & 0 & 0 \\
\hline 0 & 0 & \ldots & 0 & 0 & 1 \\
0 & 0 & \ldots & 0 & 1 & 0
\end{array}\right) .
$$

We may cap off $F^{\prime}$ with the core of the 2-handle to form a closed surface $\Sigma$ embedded in $\bar{W}$ with genus $g=\left(b_{1}(F)-1\right) / 2$, homology class $\bar{\gamma}_{0}+\sum_{i=1}^{j} 2 a_{i} \bar{\gamma}_{i}+\sum_{i=j+1}^{n}\left(2 a_{i}+1\right) \bar{\gamma}_{i}+$ $2 \alpha+b \beta$, and the self-intersection number

$$
m=-1-\sum_{i=1}^{j} 4 a_{i}^{2}-\sum_{i=j+1}^{n}\left(2 a_{i}+1\right)^{2}+4 b=e(F)+1>0 .
$$

We next choose a $\operatorname{Spin}^{c}$ structure on $W$. Since $H^{2}(\bar{W} ; \mathbf{Z}) \cong \mathbf{Z}^{n+3}$ has no 2-torsion, $\operatorname{Spin}^{c}$ structures on $\bar{W}$ are distinguished by their first Chern classes. Fix a $\operatorname{Spin}^{c} \operatorname{structure}_{\mathfrak{s}_{t}}$ on $\bar{W}$ satisfying

$$
P D\left(c_{1}\left(\mathfrak{s}_{t}\right)\right)=\varepsilon \bar{\gamma}_{0}+\sum_{i=1}^{n}\left(2 a_{i}+1\right) \bar{\gamma}_{i}+2 \alpha+2 x \beta
$$


where

$$
x=\frac{\sum_{i=1}^{j} 2 a_{i}+2(b-g)-1+\varepsilon}{4}
$$

and $\varepsilon \in\{1,-1\}$ is chosen so as to make $x$ an integer. Since the given vector is characteristic for $Q_{\bar{W}}$, it corresponds to a $\operatorname{Spin}^{c}$ structure. Furthermore, $\left\langle c_{1}\left(\mathfrak{s}_{t}\right),[\Sigma]\right\rangle=m-2 g=e(F)-$ $\beta_{1}(F)+2>0$. Applying Lemma 2 to the pair $\left(\bar{W}, S_{-1}^{3}(K)\right)$, we have

$$
c_{1}\left(\mathfrak{s}_{t}\right)^{2}+\beta_{2}^{-}(\bar{W}) \leq 1+4 d\left(S_{-1}^{3}(K)\right) .
$$

Since $c_{1}\left(\mathfrak{s}_{t}\right)^{2}=-1-\sum_{i=1}^{n}\left(2 a_{i}+1\right)^{2}+8 x=e(F)-j-1+2 \varepsilon-4 g$, the inequality (4) implies

$$
(e(F)-j-1+2 \varepsilon-4 g)+(n+2) \leq 1+4 d\left(S_{-1}^{3}(K)\right) .
$$

By using $-1 \leq \varepsilon, j \leq n$, and $2 g=\beta_{1}(F)-1$, the inequality (5) reduces to the following inequality

$$
\frac{e(F)}{2}-2 d\left(S_{-1}^{3}(K)\right) \leq \beta_{1}(F)
$$

Finally, we consider the case where $\beta_{1}(F)$ is even. Taking the connected sum of $F \subset$ $\operatorname{punc}\left(n \overline{\mathbf{C} P^{2}}\right)$ and the standard embedding of $\mathbf{R} P^{2} \subset S^{4}$ whose normal Euler number is +2 , we have a non-orientable embedded surface $\hat{F} \subset \operatorname{punc}\left(n \overline{\mathbf{C} P^{2}}\right)$ with boundary $K$ such that $\beta_{1}(\hat{F})=\beta_{1}(F)+1$ and $e(\hat{F})=e(F)+2$. Since $\beta_{1}(\hat{F})$ is odd, $\hat{F}$ satisfies the inequality (6). Hence we have

$$
\frac{(e(F)+2)}{2}-2 d\left(S_{-1}^{3}(K)\right) \leq \beta_{1}(F)+1,
$$

and this inequality (7) is equivalent to the inequality claimed in Proposition 2.

This completes the proof of Proposition 2.

\section{Proof of Theorem 3}

In this section, we prove Theorem 3 by using Proposition 2 and the following theorem.

TheOrem 5 ([10]). Let $X$ be a closed 4-manifold and $K \subset \partial($ punc $X)$ a knot. If $K$ bounds a non-orientable embedded surface $F$ in punc $X$ that represents zero in $\mathrm{H}_{2}\left(\operatorname{punc} X, \partial(\operatorname{punc} X) ; \mathbf{Z}_{2}\right)$, then

$$
\left|\sigma(K)+\sigma(X)-\frac{e(F)}{2}\right| \leq \beta_{2}(X)+\beta_{1}(F),
$$

where $\sigma(X)$ is the signature of $X$. 
By reversing the orientation of $X$, we obtain the following lemma. We also use this lemma to prove Theorem 3.

LEMMA 3. For any 4-manifold $X$ and any knot $K$, the following equality holds:

$$
\gamma_{X}^{0}(K)=\gamma_{-X}^{0}\left(K^{*}\right)
$$

where $K^{*}$ denotes the mirror image of $K$.

Let us prove Theorem 3.

Proof of Theorem 3. Let $F \subset \operatorname{punc}\left(n \overline{\mathbf{C} P^{2}}\right)$ be a non-orientable surface with boundary $K$ which represents zero in $H_{2}\left(\operatorname{punc}\left(n \overline{\mathbf{C} P^{2}}\right), \partial\left(\operatorname{punc}\left(n \overline{\mathbf{C} P^{2}}\right)\right) ; \mathbf{Z}_{2}\right)$. It follows from Theorem 5 that

$$
\left|\sigma(K)+(-n)-\frac{e(F)}{2}\right| \leq n+\beta_{1}(F) .
$$

Hence we have

$$
\beta_{1}(F) \geq \sigma(K)-\frac{e(F)}{2}-2 n .
$$

Combining this inequality with Proposition 2, we have

$$
\gamma_{n \overline{\mathbf{C} P^{2}}}^{0}(K) \geq \frac{\sigma(K)}{2}-d\left(S_{-1}^{3}(K)\right)-n .
$$

By using this inequality and Lemma 3, it follows that for any knot $K \subset \partial\left(\operatorname{punc}\left(n \mathbf{C} P^{2}\right)\right)$,

$$
\gamma_{n \mathbf{C} P^{2}}^{0}(K)=\gamma_{n \overline{\mathbf{C} P^{2}}}^{0}\left(K^{*}\right) \geq \frac{\sigma\left(K^{*}\right)}{2}-d\left(S_{-1}^{3}\left(K^{*}\right)\right)-n=\frac{-\sigma(K)}{2}+d\left(S_{1}^{3}(K)\right)-n .
$$

This proves Theorem 3 .

\section{Proof of Theorem 2}

To prove the existence of $K$ with $\gamma_{n \mathbf{C} P^{2}}^{0}(K)=k$, we take the connected sum of $n+k$ copies of $9_{42}$ for any positive integer $k$, where $9_{42}$ is the knot defined in the Rolfsen knot table [7]. Notice that $d\left(S_{1}^{3}(\cdot)\right)$ is a knot concordance invariant but not a homomorphism from the knot concordance group to integers as mentioned in [6].

Proposition 3. We have $\left.d\left(S_{1}^{3} \stackrel{m}{\# 9_{42}}\right)\right)=0$ for any positive integer $m$.

PROOF. Let $p$ be a sufficiently large integer. From the formula (1) and Proposition 1, we obtain the $d$-invariant $d\left(S_{1}^{3} \stackrel{m}{\left(\# 9_{42}\right)}\right)$ by calculating the homology of $C F K^{\infty} \stackrel{m}{\left(\# 9_{42}\right)}\{i \geq$ 0 or $j \geq 0\}$.

First, we consider the $m=1$ case. For the generators $\left\{x_{i}\right\}_{1 \leq i \leq 9}$ of $C F K^{\infty}\left(9_{42}\right)$, we use the same generators as those in Fig.14 in [5] (see Figure 3). Let $S_{1}$ denote $\left\{x_{i} \mid 1 \leq i \leq 9\right\}$. Let 


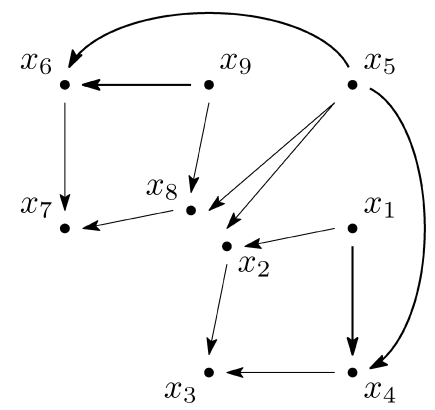

Figure 3. The differential maps of $G$ in $C F K^{\infty}\left(9_{42}\right)$. See Fig. 14 in [5].

$G$ be the differential $\mathbf{F}$-module generated by $S_{1}$, namely, $\mathbf{F}\left\langle x \mid x \in S_{1}\right\rangle$ with $\operatorname{gr}\left(x_{5}\right)=0$, where gr is the absolute grading on the chain complex $C F K^{\infty}$. The chain complex $C F K^{\infty}\left(9_{42}\right)$ consists of a differential $\mathbf{F}[U]$-module $G\left[U, U^{-1}\right]:=G \otimes_{\mathbf{F}} \mathbf{F}\left[U, U^{-1}\right]$. The generators of $H_{*}\left(C F K^{\infty}\left(9_{42}\right)\right)$ are $\left\{U^{-i} \cdot \alpha\right\}_{i \in \mathbf{Z}}$, where $\alpha=x_{1}+x_{5}+x_{9}$. The homology of the quotient complex $C F K^{\infty}\left(9_{42}\right)\{i \geq 0$ or $j \geq 0\}$ is as follows:

$$
H_{*}\left(C F K^{\infty}\left(9_{42}\right)\{i \geq 0 \text { or } j \geq 0\}\right) \cong \mathbf{F}\left\langle U^{-i} \alpha \mid i \geq 0\right\rangle \cong \mathcal{T}_{(0)}^{+} .
$$

In fact, it follows from the grading of $\alpha$ that the minimal grading of this homology is zero. Hence, in particular, $d\left(S_{1}^{3}\left(9_{42}\right)\right)=\tilde{d}\left(S_{p}^{3}\left(9_{42},[0]\right)\right)=0$.

Next, we compute $d\left(S_{1}^{3}\left(\stackrel{m}{\# 9_{42}}\right)\right.$ ). From the Künneth type formula of the Heegaard Floer homology we have $C F K^{\infty} \stackrel{m}{\left(\# 9_{42}\right)} \cong \bigotimes^{m} C F K^{\infty}\left(9_{42}\right)$. We denote the set of generators by $S_{m}=\left\{x_{i_{1}} \otimes x_{i_{2}} \otimes \cdots \otimes x_{i_{m}} \mid 1 \leq i_{k} \leq 9\right\}$ and the vector space generated by $S_{m}$ by $G_{m}=\mathbf{F}\left\langle x \mid x \in S_{m}\right\rangle$, where $\operatorname{gr}\left(x_{5}^{\otimes m}\right)=0$. Here let $y^{\otimes m}$ denote the $m$-th tensor product $y \otimes \cdots \otimes y$.

The chain complex $\left.C F K^{\infty} \stackrel{m}{\#} 9_{42}\right)$ is the summation

$$
\bigoplus_{i \in \mathbf{Z}}\left(U^{-i} \cdot G_{m}\right)=\mathbf{F}\left[U, U^{-1}\right] \otimes_{\mathbf{F}} G_{m}=: G_{m}\left[U, U^{-1}\right] .
$$

Hence, we may consider each homology $H_{*}\left(U^{-i} \cdot G_{m}\{i \geq 0\right.$ or $\left.j \geq 0\}\right)$.

The differential $\partial^{\infty}$ in $\otimes^{m} C F K^{\infty}\left(9_{42}\right)$ is computed as follows:

$$
\partial^{\infty}\left(z_{1} \otimes \cdots \otimes z_{m}\right)=\sum_{k=1}^{m} z_{1} \otimes \cdots \otimes \partial^{\infty} z_{k} \otimes \cdots \otimes z_{m} .
$$

By using this definition, we have $\partial^{\infty}\left(U^{-l} \cdot \alpha^{\otimes m}\right)=0$. Since $U^{-l} \cdot \alpha^{\otimes m}$ has the unique top grading in $U^{-l} \cdot G_{m}$, we have $\alpha^{\otimes m} \notin \operatorname{Im}\left(\partial^{\infty}\right)$. Hence the generator of $H_{*}\left(U^{-l} \cdot G_{m}\right)$ is 


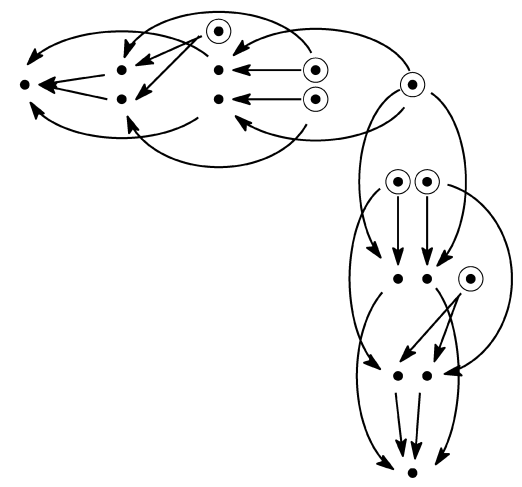

FIGURE 4. The chain complex $G_{2}\{i \geq 0$ or $j \geq 0\}$ and the homological generator

$$
\left(x_{5}+x_{9}\right)^{\otimes 2}+\left(x_{5}+x_{1}\right)^{\otimes 2}+x_{5}^{\otimes 2} \text {. }
$$

$U^{-l} \cdot \alpha^{\otimes m}$

For the case where $l<0$, since the generators in $U^{-l} \cdot G_{m}$ are in $C F K^{\infty} \stackrel{m}{\left(\# 9_{42}\right)}\{i<$ 0 and $j<0\}$, the minimal degree of $\mathcal{T}^{+}$-component in $C F K^{\infty} \stackrel{m}{\left(\# 9_{42}\right)}\{i \geq 0$ or $j \geq 0\}$ is non-negative.

We consider the component of $l=0$. Let $\varphi$ denote the natural isomorphism:

$$
\varphi: G_{m} /\left(G_{m}\{i<0 \text { and } j<0\}\right) \cong G_{m}\{i \geq 0 \text { or } j \geq 0\} .
$$

LEMMA 4. The map $\varphi$ satisfies the following:

$$
\varphi\left(\alpha^{\otimes m}\right)=\left(x_{5}+x_{9}\right)^{\otimes m}+\left(x_{5}+x_{1}\right)^{\otimes m}+x_{5}^{\otimes m} .
$$

PROOF OF LEMMA 4. Expanding $\alpha^{\otimes m}$, we have

$$
\alpha^{\otimes m}=\sum_{i_{j} \in\{1,5,9\}} x_{i_{1}} \otimes \cdots \otimes x_{i_{m}} .
$$

If the set $\left\{i_{1}, \ldots, i_{m}\right\}$ of the suffixes of each term in the summation above contains $\{1,9\}$, then the $(i, j)$-coordinate must have $i<0$ and $j<0$. Conversely, if the $(i, j)$-coordinate of $x_{i_{1}} \otimes$ $\cdots \otimes x_{i_{m}}$ has $i<0$ and $j<0$, then the set $\left\{i_{1}, \ldots, i_{m}\right\}$ must contain $\{1,9\}$. The whole sum of the terms $x_{i_{1}} \otimes \ldots \otimes x_{i_{m}}$ satisfying $\{1,9\} \not \subset\left\{i_{1}, \ldots, i_{m}\right\}$ is $\left(x_{5}+x_{9}\right)^{\otimes m}+\left(x_{5}+x_{1}\right)^{\otimes m}+x_{5}^{\otimes m}$. Therefore, the assertion claimed in Lemma 4 follows.

Here, as an example, we describe the boundary maps in $G_{2}\{i \geq 0$ or $j \geq 0\}$ in Figure 4.

The term $\varphi\left(\alpha^{\otimes m}\right)$ is a generator in $H_{*}\left(G_{m}\{i \geq 0\right.$ or $\left.j \geq 0\}\right)$, because $\partial^{\infty}\left(\varphi\left(\alpha^{\otimes m}\right)\right)=$ $\varphi\left(\partial^{\infty}\left(\alpha^{\otimes m}\right)\right)=0$ and the element $\varphi\left(\alpha^{\otimes m}\right)$ has the top grading in $G_{m}\{i \geq 0$ or $j \geq 0\}$. The image $\varphi\left(\alpha^{\otimes m}\right)$ is in the $\mathcal{T}^{+}$-component with the minimal grading, because the whole 

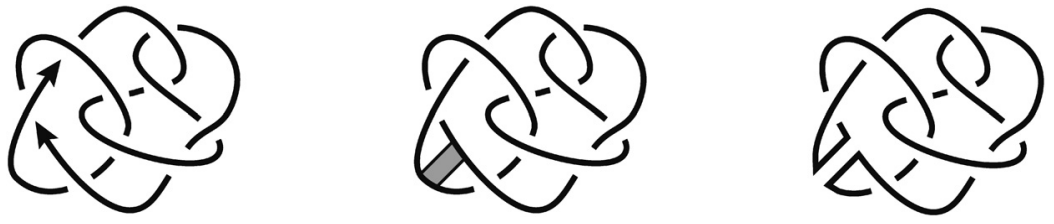

FIgURE 5. The knot $9_{42}$ bounds an embedded Möbius band in $B^{4}$.

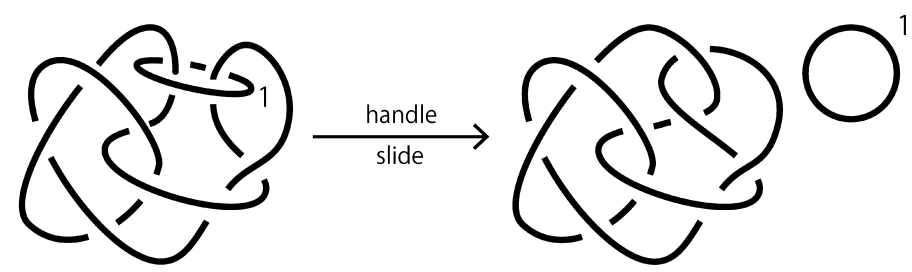

FIGURE 6. The knot $9_{42}$ bounds an embedded disk in punc $\mathbf{C} P^{2}$.

chain complex $C F K^{\infty} \stackrel{m}{\left(\# 9_{42}\right)}$ is generated by $\left\{U^{-l} \cdot \alpha^{\otimes m} \mid l \in \mathbf{Z}\right\}$. The minimal degree of $G_{m}\{i \geq 0$ or $j \geq 0\}$ is $\operatorname{gr}\left(\varphi\left(\alpha^{\otimes m}\right)\right)=\operatorname{gr}\left(\alpha^{\otimes m}\right)=0$. This means

$$
\left.\left.d\left(S_{1}^{3} \stackrel{m}{\# 9_{42}}\right)\right)=\tilde{d}\left(S_{p}^{3} \stackrel{m}{\# 9_{42}},[0]\right)\right)=0 .
$$

Actually, $\left(x_{5}+x_{9}\right)^{\otimes m}+\left(x_{5}+x_{1}\right)^{\otimes m}+x_{5}^{\otimes m}$ is the unique generator in $H_{*}\left(G_{m}\{i \geq\right.$ 0 and $j \geq 0\}$ ). This fact is not needed here, and we skip the proof.

Proof of TheOREM 2. Since $\sigma\left(9_{42}\right)=-2$ and the knot signature is additive, we have $\sigma\left(\stackrel{n+k}{\#} 9_{42}\right)=-2(n+k)$. Thus, by using Theorem 3 and Proposition 3 , we have

$$
\left.\gamma_{n \mathbf{C} P^{2}}^{0} \stackrel{n+k}{\#} 9_{42}\right) \geq \frac{-(-2(n+k))}{2}+0-n=k \text {. }
$$

We next construct a non-orientable embedded surface $F_{n, k} \subset \operatorname{punc}\left(n \mathbf{C} P^{2}\right)$ satisfying the following:

1. $\partial F_{n, k}=\stackrel{n+k}{\#} 9_{42}$,

2. $\beta_{1}\left(F_{n, k}\right)=k$, and

3. $F_{n, k}$ represents zero in $H_{2}\left(\operatorname{punc}\left(n \mathbf{C} P^{2}\right), \partial\left(\operatorname{punc}\left(n \mathbf{C} P^{2}\right)\right) ; \mathbf{Z}_{2}\right)$.

The cobordisms in Figure 5 and 6 give a properly embedded Möbius band $M$ in $B^{4}$ with boundary $9_{42}$, and a properly embedded disk $D$ in punc $\mathbf{C} P^{2}$ with boundary $9_{42}$ which represents zero in $H_{2}$ (punc $\mathbf{C} P^{2}, \partial\left(\right.$ punc $\left.\mathbf{C} P^{2}\right) ; \mathbf{Z}_{2}$ ). Taking the boundary connected sum of $n$ 
copies of (punc $\left.\mathbf{C} P^{2}, D\right)$ and $k$ copies of $\left(B^{4}, M\right)$, we have a new non-orientable embedded surface $F_{n, k}$ satisfying the above properties from (1) to (3). This completes the proof.

ACKNOWLEDGMENT. The first author was supported by JSPS KAKENHI Grant Number 15J10597. The second author was supported by JSPS KAKENHI Grant Number 24840006. The authors would like to thank Kokoro Tanaka and Akira Yasuhara for their useful comments and encouragement.

\section{References}

[ 1 ] J. BATson, Nonorientable four-ball genus can be arbitrarily large, Math. Res. Lett. 21 (2014), no. 3, $423-436$.

[ 2 ] P. M. Gilmer and C. Livingston, The nonorientable 4-genus of knots, J. Lond. Math. Soc. (2) 84 (2011), 559-577.

[ 3 ] R. A. Norman, Dehn's lemma for certain 4-manifolds, Invent. Math. 7 (1969), 143-147.

[ 4 ] P. OZSVÁth and Z. SZABÓ, Absolutely graded Floer homologies and intersection forms for four-manifolds with boundary, Adv. Math. 173 (2003), 179-261.

[ 5 ] P. OzsvátH and Z. SZABÓ, Holomorphic disks and knot invariants, Adv. Math. 186 (2004), 58-116.

[6] T. D. PETERS, A concordance invariant from the Floer homology of \pm 1 surgeries, arXiv:1003.3038 (2010).

[ 7 ] D. Rolfsen, Knots and links, Mathematics Lecture Series, No. 7. Publish or Perish, Inc., Berkeley, Calif., 1976. MR 58:24236; corrected reprint MR 95c:57018.

[ 8 ] S. SuZuKI, Local knots of 2-sphere in 4-manifolds, Proc. Japan Acad. 45 (1969), 34-38.

[9] O. VIRO, Positioning in codimension 2, and the boundary, Uspehi Mat. Nauk 30 (1975), 231-232.

[10] A. Yasuhara, Connecting lemmas and representing homology classes of simply connected 4-manifolds, Tokyo J. Math. 19 (1996), 245-261.

Present Addresses:

KOUKI SATO

DEPARTMENT OF MATHEMATICS,

TOKYO InSTITUTE OF TECHNOLOGY,

2-12-1 OoKayama, Meguro-KU,

TOKYO 152-8551, JAPAN.

e-mail: sato.k.bs@m.titech.ac.jp

Motoo TAnge

INSTITUTE OF MATHEMATICS,

UNIVERSITY OF TSUKUBA,

IBARAKI 305-8571, JAPAN.

e-mail: tange@math.tsukuba.ac.jp 